\title{
EAl Endorsed Transactions

\section{Web interactive non intrusive load disaggregation system for active demand in smart grids}

\author{
G.M.Tina ${ }^{1}$, V. A. Amenta ${ }^{2}$, O. Tomarchio ${ }^{3}$, G. Di Modica ${ }^{4}$ \\ ${ }^{1}$ giuseppe.tina@dieei.unict.it, ${ }^{2}$ valeria.amenta@dieei.unict.it, ${ }^{3}$ orazio.tomarchio@dieei.unict.it, ${ }^{4}$ gdimodica@ diit.unict.it
}

\section{Abstract}

A Smart Grid combines the use of traditional technology with innovative digital solutions, making the management of the electricity grid more flexible. It allows for monitoring, analysis, control and communication within the supply chain to improve efficiency, reduce the energy consumption and cost, and maximize the transparency and reliability of the energy supply chain. The optimization of energy consumption in Smart Grids is possible by using an innovative system based on Non Intrusive Appliance Load Monitoring (NIALM) algorithms, in which individual appliance power consumption information is disaggregated from single-point measurements, that provide a feedback in such a way to make energy more visible and more amenable to understanding and control. We contribute with an approach for monitoring consumption of electric power in households based on both a NILM algorithm, that uses a simple load signatures, and a web interactive systems that allows an active role played by users.

Keywords: Smart grid, load monitoring; NIALMS; energy management; Feedback, ICT.

Received on 15 August 2014, accepted on 02 September 2014, published on 12 December 2014

Copyright $\odot 2014$ G.M. Tina et al., licensed to ICST. This is an open access article distributed under the terms of the Creative

Commons Attribution licence (http://creativecommons.org/licenses/by/3.0/), which permits unlimited use, distribution and reproduction in any medium so long as the original work is properly cited.

doi: 10.4108/ew.1.3.e4

\section{Introduction}

Nowadays the need to both solve environmental problems and cope with the exhausting of traditional fossils has forced many countries and organizations (e.g. the European Community) to put in their political agenda the energy problem. In this context to find a sustainable solution it is needed to combine three different strategies: energy saving, efficiency and renewable energies. On the other hand the great development of ICT technologies has drawn the attention of many researchers on the development of smart grids as a hardware and software structure that can allow the actuation of the energetic strategies. Smart grids are so complex and varied that a unique definition is needed.

The Organization for Economic Cooperation and Development (OECD) beholds Smart Grid in two perspectives [1]. From a solution perspective, the smart grid is characterized by:

- More efficient energy routing and thus an optimized energy usage, a reduction of the need of excess capacity and increased power quality and security.
- Better monitoring and control of energy and grid components. Improved data capture and thus an improved outage management.

- Two-way flow of electricity and real-time information allowing for the incorporation of green energy sources, demand-side management and time market transactions.

- Highly automated, responsive and selfhealing energy network with seamless interfaces between all parts of the grid;

From a technical components' perspective, the main components of a smart grid are:

- New and advanced grid components.

- Smart devices and smart metering.

- Integrated communication technologies.

- Decision support systems and human interfaces.

- Advanced control systems.

In this continuously evolving system the user becomes the protagonist through the use of electronic devices which makes transparent consumption, encourages his active participation in the energy market, promotes a rational use of energy. 
Commercial solutions to improve the management of energy demand have centered on the deployment of smart meters and in-home energy displays that can provide whole-house real-time energy monitoring and dynamic pricing from suppliers in order to motivate users to shift or reduce their energy consumption [2].

A number of countries and regions are deploying new electricity metering, and its introduction is being accelerated by legislation: in the European Union the $80 \%$ of households will have a smart meter by 2020 . Austria and Switzerland commissioned a bi-national study on the topic "Smart Metering Consumption" [14]. The study, completed in 2012, assessed the energy used by existing and planned metering, to better understand the impact of implementing large scale roll out of smart metering, and to estimate the own energy consumption required for the operation of this infrastructure. In 2012 study on Smart Metering Infrastructure (SMI) was completed and presented by Austria and Switzerland to the 4E ExCo as well. The study included two major topics namely, Smart Metering Consumption (SMC) and Non Intrusive Load Monitoring System (NIALM). The term NILM is sometimes also used for NIALM.

The domestic energy monitoring infrastructure planned today will be set for decades, millions of smart meters will be deployed, and the associated energy consumption will be fixed with the technology and architecture chosen for these systems. However, end-users at household level have often been excluded from this energy efficiency optimization process: they have traditionally held a passive role in issues related to energy savings although it rests on them to decide the amount of energy to consume and how to utilize it. As a result, it is quite evident that consumers also need to be active players in this process and research suggests that users are willing and capable to adapt their behaviour to energy saving practices if the necessary feedback, support and incentives are given [3]. The increasing concern about the impact of energy usage on the environment as well as the rise of energy costs are arguably the main factors that encourage customers to look for ways of decreasing consumes.

Nevertheless, the major difficulty is the lack of information about day-to-day activities; for instance, energy bills, which are usually received at the end of each month, cannot be used to distinguish the effects of individual actions or to obtain meaningful feedback about the effectiveness of users' change of habits [4][5]. Such problems need innovative feedback mechanisms with greater transparency about the consumption at any time and the associated cost that can potentially improve energy savings practices. Current trends in the development and convergence of ICT and energy networks are ushering a range of possibilities in areas such as residential energy monitoring (measuring, processing and providing feedback in near-real-time), context-aware application and activity detection [6].

In this paper we present an overall ICT architecture for energy consumption awareness: data about energy consumption collected in users' homes are sent to a service provider site that, after disaggregating and processing them, allows a user friendly representation of energy consumption providing the user with a direct feedback about his habits and distribution of consumption among his appliances.

Within this architecture, one important software module is related to a Non Intrusive Appliance Load Monitoring (NIALM) algorithm, in which individual appliance power consumption information is disaggregated from singlepoint measurements, that provide a feedback in such a way to make energy more visible and more amenable to understanding and control. One of the main contribution of this work is a new and simple algorithm for disaggregating the overall consumption into individual appliance. The rest of the paper is organized in the following way. Section II describes the overall system architecture. In Section III basic NIALM concepts are presented. In section IV numerical analysis of experimental results are discussed. Then in section $\mathrm{V}$, the SEEE project along with on field experimental tests are presented. Finally we draw some conclusions and propose future development in Section VI.

\section{System architecture}

In this Section the overall architecture of the system is presented. In the envisaged scenario, energy consumption data coming from users' homes are sent to a service provider where data are appropriately processed in order to give users detailed information about their energy consumption habits. The overall architecture, shown in Figure 1, is broken down into three main subsystems:

- Local monitoring subsystem: it is composed of a power meter installed at the user's home, where it measures the global energy consumption of the user (along with other energy parameters) and a network device able to communicate the measured data to the central monitoring subsystem by means of a common Internet link.

- Central monitoring subsystem: it is the core of the system, where all the processing takes place. It has all the software components required to process the data coming from the power meters, to execute the NIALM algorithms, to store processed data on an appropriate DBMS, and to generate a graphical data presentation for the end user.

- User presentation: being typically a web-based interaction, it does not require any special prerequisites on the user side allowing, among other things, to access the system wherever they may be (after a suitable authentication). 


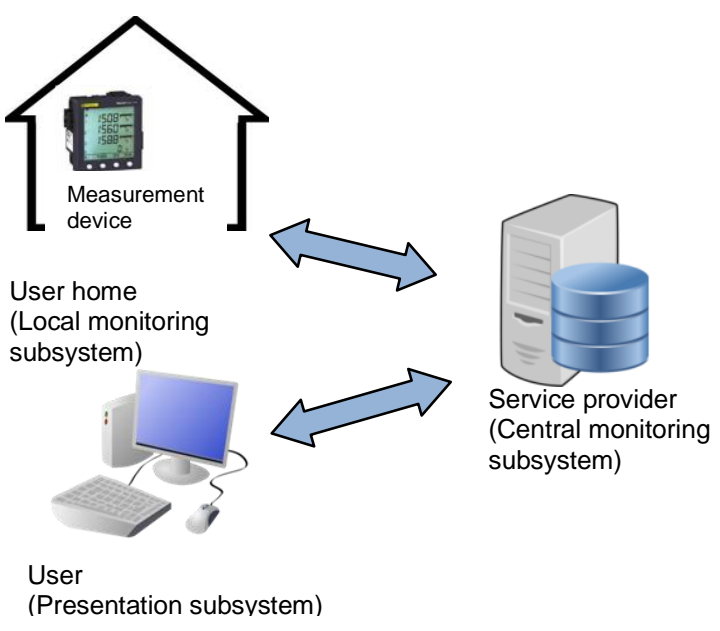

Figure 1 Overall system architecture

The logical architecture of the Central monitoring subsystem is represented in Figure 2

It is composed of several modules providing the following features:

- Data communication: it is the module that manages the communication with the Local monitoring systems and deals with the store of measured data into the Raw DB. The protocol used to get the measured data is the Modbus/TCP, which allows to directly query the measurement device over an IP network.

- NIALM module: by means of a novel NIALM algorithm, it is able to perform a workload characterization that disaggregates the global energy consumption of the appliances. Details on the behavior and the algorithm executed by this module will be given in section III.

- Data Management and Persistence: it manages data persistence and controls data access for the purpose of historical data analysis and presentation to the end user. Three different databases are used:

- Raw $D B$ : it stores the raw data coming from the local monitoring systems; these are the input data for the NIALM module;

- Processed DB: it stores the results produced by the NIALM module; it also contains intermediary information generated by the system's business logic;

- User configuration management: this module contains users related data, such as information about their appliances and their configurations, users' feedback, and so on.

- Business logic: it is the module containing the logic needed to process data and generate the useful information to be presented to the home user;

- Presentation layer: this layer is responsible for presenting the home user with the required information. It is equipped with a simple and effective web interface. Through the web interface users may also provide "feedbacks" regarding their consumption habit, thus proactively interacting with the system in order to improve the behavior of the NIALM algorithm.

- Billing system interaction: this module manages the interaction with the billing system of the energy provider. It retrieves information about the provider's cost of energy and transforms the users' data related to energy consumption (measured in Watt) into an actual cost.

A simplified view of the information model used within the software architecture is represented in UML class diagram depicted in Figure 3.

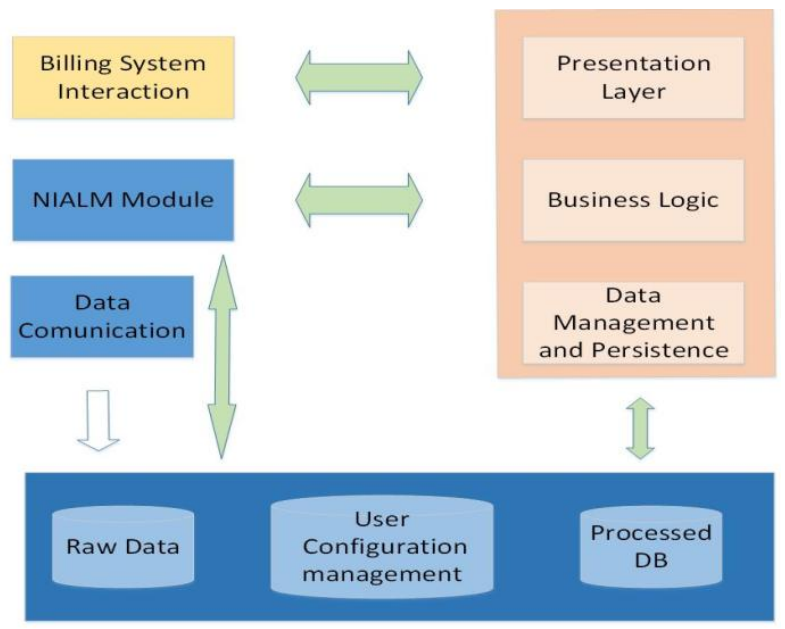

Figure 2 Software architecture

In the diagram entities, mutual relationships and relationships' cardinalities were modelled; entities-roles are briefly explained in the following:

- User: it models the information associated to the user (authentication information included).

- Power Meter: this entity models the measurement device installed at the user's home.

- Device: this entity models a generic device consuming energy (appliance). It is characterized by some energy parameters that constitute its signature load.

- Category: devices are grouped into categories, in order to allow for simple filtering and compact visualization by users.

- Device energy consumption: this entity stores all the consumption data associated to a device obtained from the NIALM algorithm.

- State: it represents the state of the device. 
- Global raw data: this entity is needed to store the global consumption data and associated measurements produced by the Power Meter and locally stored into the RawDB.

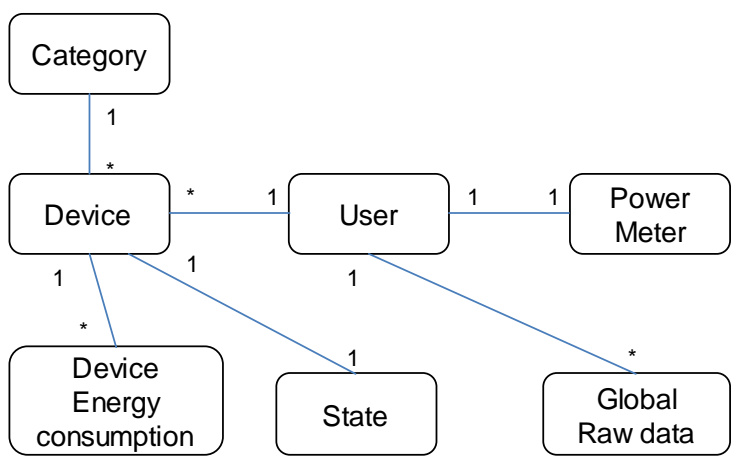

Figure 3 Information Model

\section{The NIALM concept}

Non-intrusive methods are intended to offer installation simplicity and the ability to distinguish important load changes measurements at a central monitoring point [7]. Researchers at MIT were the early users of this technology to monitor residential and commercial enduser loads [8].

In the proposed method, the operating states of given appliance are determined by identifying moments where its active and/or reactive power consumption measurements change from one nearly constant or steadystate value to another one. These steady-state changes usually correspond to the appliance either turning on or turning off and they are characterized by a magnitude value and a sign (in active and/or reactive power $( \pm \Delta \mathrm{P}$, $\pm \Delta \mathrm{Q})$ ).

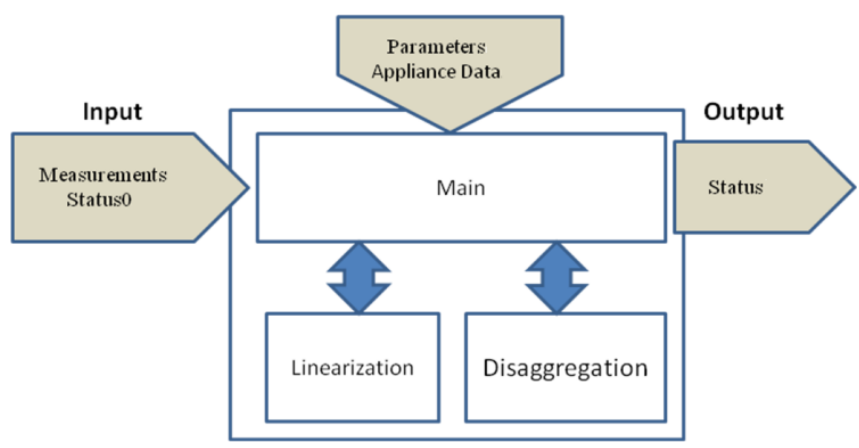

Figure 4 Overview diagram of the NILM algorithm

In Figure 4 the main structure of the NILM algorithm is presented. The output is the status of each appliance, whereas the input can be classified as follows:

- Measurements: it contains the information coming from the local monitoring subsystem (e.g. voltage, current, active and reactive power, total harmonic distortion and so on).
- Status0: it is the daily initial status of all appliances.

- Appliance Data: it contains information about type and signature of loads, mainly rated active (P) and reactive (Q) power. It is worth mentioning that the initial information about loads can be obtained only from data in nameplates or in technical documentation provided by the manufacturer.

- Parameters: the NILM algorithm needs some parameters that are somehow correlated with the set of appliances.

The NILM program is written in the Matlab programming language and its behavior is described by the flowchart depicted in [9]. Measurements have to be preprocessed before entering the NILM algorithm: this data treatment aims to smooth out small or erratic variations in the total demand signal. This preprocessing consists mainly in $\mathrm{P}$ and $\mathrm{Q}$ linearization. It performs also filtering of spikes and low frequency noise. Filtered signals consist of piecewise line where each positive or negative demand drop is more likely to represent a significant ON or OFF signal. The algorithm compares each change in the global $\mathrm{P}$ signal to each rated power appliance. If the magnitude of the change is greater than the rated power of an appliance the change is attributed to that appliance. If there are more than one candidate that can generate the same power drop, a new comparison in change in the Q global signal is applied. So as matter of fact, the proposed algorithm uses only power analysis to characterize the signature of electric appliances.

Although this method achieves an estimated detection accuracy of about $70 \%$, it is possible to improve these results by an effective interaction with customers.

\section{Numerical Results}

The robustness of the disaggregation algorithm has been tested both numerically and experimentally. Specifically, in this section, the function, that randomly generates different and controlled load profiles is described. The main parameters that need to be set to generate a load demand profile are: the number of the appliances $\left(\mathrm{n}_{\mathrm{a}}\right)$ and the number of samples $\left(\mathrm{n}_{\mathrm{s}}\right)$, as well as deltaT, which is the minimum number of samples between a switch on and a switch off of an appliance. In this numerical analysis these parameters assume the following values: $\mathrm{n}_{\mathrm{a}}=10, \mathrm{n}_{\mathrm{s}}=500$ and deltaT $=2$.

For sake of simplicity, in this numerical analysis, only ON-OFF appliances are chosen, whereas in the proposed NIALM algorithm also multi-state loads are considered.

Figure 5 shows an example of a generated global load profiles, active and reactive power. 


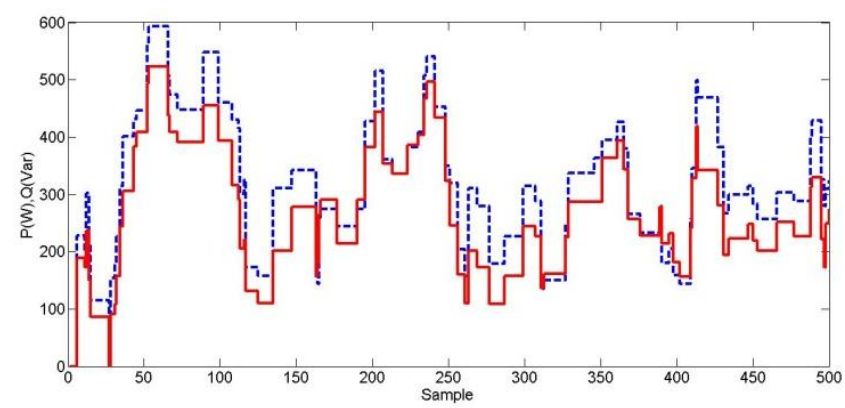

Figure 5 Global generated load profiles: active power (solid red line) and reactive power (dashed blue line)

This function also generates some data sets containing useful information to run the disaggregation algorithm. Further, the program provides the load profiles for each appliance in such a way that it will be possible to compare the profiles coming from disaggregation to the real ones.

There are many factors that significantly affect the efficiency of the disaggregation algorithm, such as: measurement accuracy of power meters (normally $\pm 1 \%$ f.s. and +-0.25 f.s.), electrical characteristics of the customer's appliances, and, finally it is related to actions that can fulfill the users.

Related to the appliances electrical characteristics there are two important aspects that impact greatly the effectiveness of disaggregation: the first one is connected with the nominal power of appliances. In fact when in a domestic dwelling, there are appliances whose rated powers are too small or close each other, considering a given power threshold (e.g. $4 \mathrm{~W}$ ), the efficiency tends to decrease. The second one is linked with the simultaneous switching on and/or off of two or more appliances.

In [12] a similar approach is adopted to evaluate the robustness of a NIALM algorithm, the following disturbs are considered: variation of the rated power of appliances (due to variation of frequency and voltage) and unknown not stored appliances.

In this context two indices that characterize a given group of appliances have been defined, that is $\mathrm{KG}_{\mathrm{P}}$ and $\mathrm{Dg}_{\mathrm{P}}$.

$K G_{P}=\frac{P_{\min }}{\text { Lim_DeltaP }}$

Where:

- $\mathrm{P}_{\min }$ is the smallest value among the rated power of appliances belonging to a given group;

- Lim_DeltaP is a parameter, i.e. $4 \mathrm{~W}$, it is a threshold for the detection of the switching events.

$D g_{P}=\min \left\{\left|P_{i}-P_{j}\right|\right\} \quad$ where $i, j \in\left[1,2, \ldots, n_{a}\right]$

$\mathrm{Dg}_{\mathrm{P}}$ is the smallest value among the values obtained from the differences between the rated power of $i$-th and $j$-th appliance. Similar indices have been defined for the reactive power, $\mathrm{KG}_{\mathrm{Q}}$ and $\mathrm{Dg}_{\mathrm{Q}}$.

Finally it has been introduced another index, named global number of simultaneous switching - GNSS. Given a certain period of time, for example a day, it is equal to the number of samples characterized by simultaneous switchings, $\mathrm{n}_{\mathrm{ss}}$, by the number of the involved appliances $\mathrm{n}_{\mathrm{ca}}[13]$.

$G N S S=\sum_{i=1}^{n s s} n_{c a}(i)$

A numerical calculation of $\mathrm{KG}_{\mathrm{P}}$ and $\mathrm{Dg}_{\mathrm{P}}$ has been performed referring to the load profiles shown in Figure 5 , and the results are reported in Table 1 . The value reported in red is $\mathrm{P}_{\min }$ in (1).

Table 1 Rating of appliances and global indices

\begin{tabular}{lrrr|l|l|}
\hline Appliance & \multicolumn{3}{c|}{ Rating } & \multirow{2}{*}{$\mathrm{KG}_{\mathrm{P}}$} & \multirow{2}{*}{$\operatorname{Dg}_{\mathrm{P}}$} \\
\hline & $\mathrm{P}(\mathrm{W})$ & $\mathrm{Q}(\mathrm{var})$ & $\operatorname{Cos}\left(^{\varphi}\right)$ & & \\
\hline 1 & 86 & 87.73 & 0.70 & & \\
2 & 64 & 99.75 & 0.54 & & \\
3 & 17 & 25.81 & 0.55 & & \\
4 & 25 & 15.49 & 0.85 & 4.25 & 2 \\
5 & 29 & 32.13 & 0.67 & & \\
6 & 86 & 114.16 & 0.60 & & \\
7 & 91 & 153.48 & 0.51 & & \\
8 & 50 & 46.81 & 0.73 & & \\
9 & 62 & 87.07 & 0.58 & & \\
10 & 77 & 30.43 & 0.93 & & \\
\hline TOTAL & $\mathbf{5 8 7}$ & $\mathbf{6 9 2 . 8 6}$ & $\mathbf{0 . 6 5}$ & & \\
\hline
\end{tabular}

The graphical results of the disaggregation algorithm, described in [9], are reported in Figure 6 where the global profile generated, named $\mathrm{P}_{\mathrm{gg}}$, is shown in red and the global profile coming from the application of the disaggregating algorithm, named $\mathrm{P}_{\mathrm{gd}}$, is shown in blue.

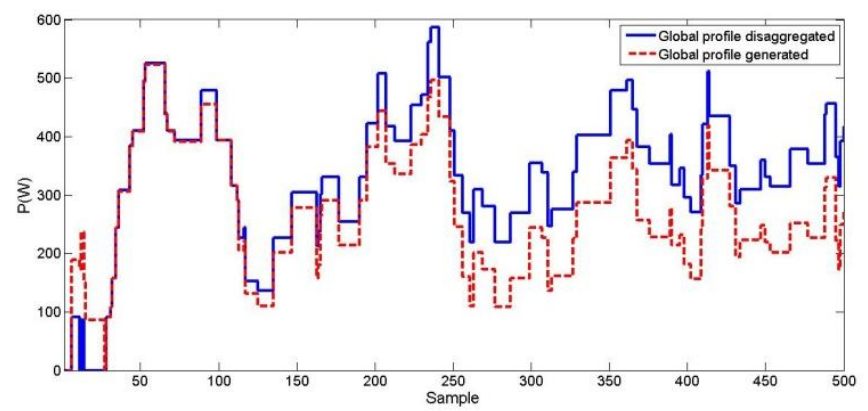

Figure 6 Global load profile Pg: Pgg- generated (dashed red line) and Pgd-disaggregated (solid blue line).

The efficiency of the NILM algorithm must be evaluated quantitatively [10]; in this context two efficiencies have been defined .

The first efficiency is based on the different, sample by sample of the generated and disaggregated global power 
[9] [11]. In (4) only the active power efficiency is shown, but the reactive power efficiency has a similar definition.

$\eta_{p}=\frac{\sum_{i=1}^{n_{s}}\left|P_{g d}(i)-P_{g g}(i)\right|}{\sum_{i=1}^{n_{s}} P_{g g}(i)}$

Where:

$\mathrm{i}$ is the $\mathrm{i}$-th sample;

$\mathrm{n}_{\mathrm{s}}$ is the number of time samples;

$\mathrm{P}_{\mathrm{gd}}$ is global disaggregated load profile;

$\mathrm{P}_{\mathrm{gg}}$ is global generated load profile.

Due to compensation phenomena it can be happen that $\eta_{p}$ can be very high (e.g $80 \% \div 90 \%$ ), but the profiles of the single appliance can be wrong. This is the reason why another efficiency, based on the state errors, has been introduced, that is $\eta_{\mathrm{s}}$, the definition is:

$\eta_{s}=\frac{\sum_{j=1}^{n_{a}}\left\{\left\{\frac{1}{n_{s}} \sum_{i=1}^{n_{s}}\left[\operatorname{xor}\left(S S A_{g, j}(i), S S A_{d, j}(i)\right)\right]\right\} * P_{j}\right\}}{\sum_{j=1}^{n_{a}} P_{j}}$

Where:

$\mathrm{j}$ is the $\mathrm{j}$-th appliance;

$\mathrm{n}_{\mathrm{a}}$ is the number of appliances;

$P_{j}$ is the rated power of $j$ th appliance

$\operatorname{SSA}_{g, j}(i)$ and $\operatorname{SSA}_{d, j}(i)$ are logical values, that indicate the states of the appliance $j$ at time $i$.

The logical operation xor allows to have a value equal to one when $\operatorname{SSA}_{\mathrm{g}, \mathrm{j}}(\mathrm{i})$ and $\mathrm{SSA}_{\mathrm{d}, \mathrm{j}}(\mathrm{i})$ assume the same values (that can be either 0 or 1 ).

To evaluate how the disaggregation algorithm works with different set of appliances, charaterized by the two indices Dgp and KGp, twenty sets of appliances have been generated and the global profiles disaggregated. The results are reported in [13], where it is shown also that a NIALM approach based only on P and Q load signatures is not sufficient to reach very high efficiency in the load disaggregation.

\section{SEEE project and on field validation tests}

A project named SEEE (Systems Efficiency for Energy Emancipation), whose partners are the department DIEEI of University of Catania, Catania (Italy) and a Sicilian energy trader, is under development; it aims to study hardware and software solutions for providing advanced tools to electric utilities users (mainly residential) not only to optimize their energy consumptions but also to make them an active part of future Smart Grids. In this way the users can have access to information and tools, in such a way to become aware of their energy consumptions habits. Further it will be possible to use information on the energy price and tariffs, and to reach many objectives such as: increasing the reshaping of load curves (i.e. redirect the electrical loads to the hours of low network usage), improving efficiency and reducing the electricity bill.

The main idea of the proposed architecture is to exploit as much as possible the commitment of the users by means of active interaction with a dedicated web site. The user plays an active roles in the whole process in many steps. Firstly, the user is required to communicate the list of appliances connected to the main power supply along with some information about their electrical characteristics.

The more complete the information provided by the user during this phase, the more accurate the results provided by the NIALM algorithm. However, since we are aware that not only the user may not be able to provide precise and complete data about his appliances, but also the results of NILM algorithm are affected by errors (see par. 4), an interactive phase has been designed. So, during the normal operation of the system, the user can be engaged in two different kinds of interactions, i.e. feedback, named respectively Check status and Verify signature, hereinafter described:

- Check status: the user is prompted to confirm some information about the status of one or more appliances. He has to provide information related to the state, (on/off) of the i-th appliance. By means of this information, the NIALM algorithm will improve, and correct, if necessary, its disaggregation results.

\begin{tabular}{|l|l|l|l|l|}
\hline $\begin{array}{l}\text { Appliance } \\
\text { name }\end{array}$ & $\begin{array}{l}\text { Status } \\
\text { from NILM }\end{array}$ & FEEDBACK (FB) I & $\begin{array}{l}\text { Appliance } \\
\text { name }\end{array}$ & $\begin{array}{l}\text { Status } \\
\text { from FB }\end{array}$ \\
\hline Name 1 & On & $\begin{array}{l}\text { Name 1 } \\
\text { On }\end{array}$ \\
\hline Name 2 & Off & Name 2 & Off \\
\hline Name $\mathrm{n}-1$ & Off & On & Is the i-th appliance \\
on or off ? & Name n-1 Off \\
\hline Name n On & & Name n & On \\
\hline
\end{tabular}

Figure 7 Logic scheme of check status feedback

- Verify signature: The user is asked to turn on and/or turn off a specific appliance, in order to revise the signature of the $\mathrm{i}$-th load. This interaction is enforced if the user, during the preliminary step, has not been able to provide all the needed information related to nominal power of appliances. So the rated power of ith appliance is corrected from P and Q to P' and Q'. 


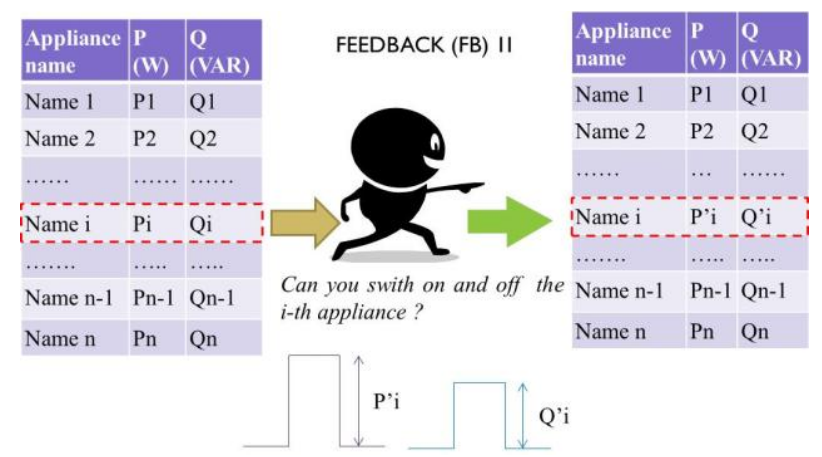

Figure 8 Logic scheme of verify status feedback

The presentation layer has been implemented as a Web application by following a Model-View-Controller (MVC) paradigm. While the Controller part has been implemented as a Java Servlet, the Model and the different Views have been implemented through HTML and JSP pages. The designed system has been developed in a real domestic scenario in order to better evaluate the overall behavior of the system.

The graphs, in Figure 9, Figure 10, and Figure 11 show the power consumption of a typical domestic user (e.g.user A). These pieces of information can help the user to understand his energetic behavior. Specifically Figure 9 shows the home page where the global measurements (P, Q, V, I, THD, Power factor) are shown in different time frames (one day, two days and one week), and updated every three minutes. Since data produced by the NIALM module are stored in the database (Processed DB), the user can decide what to analyze. Figure shows the "Appliance Power" page, where the NIALM algorithm results are represented by a piecewise graph with the same time features as the previous graph.

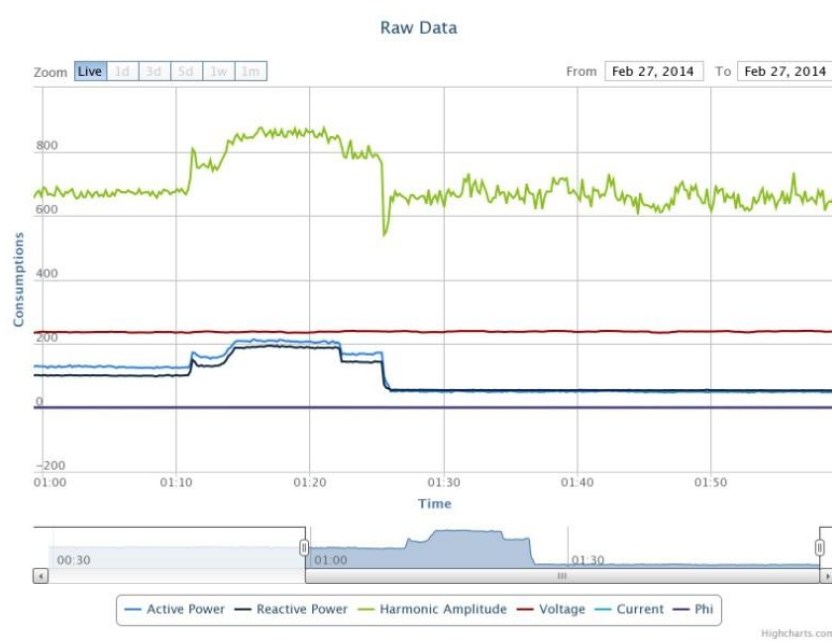

Figure 9 Home page of SEEE web site.

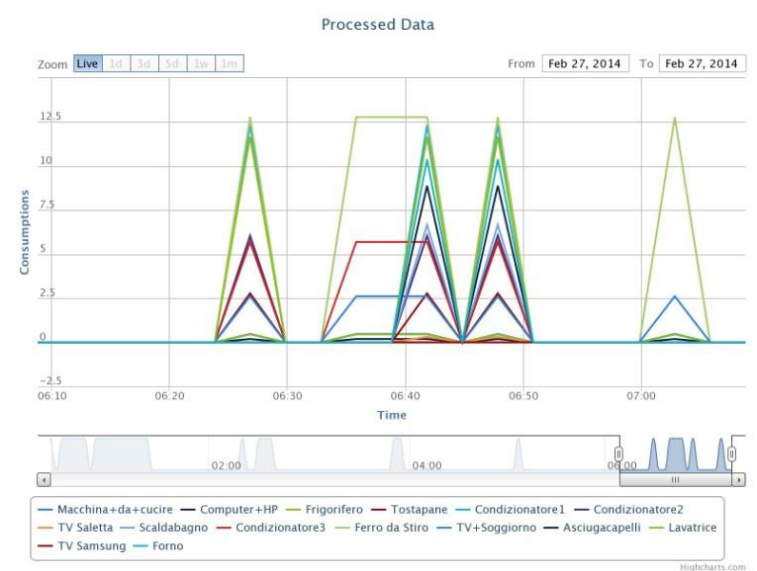

Figure 10 Power consumption grouped by appliance.

Finally, in the Overview page shown in Figure 11, two pie charts are presented: pie chart A where the monthly percentage of energy composition of each appliance is depicted, and pie chart $B$ which shows the cost rate of the electricity bill for each appliance.
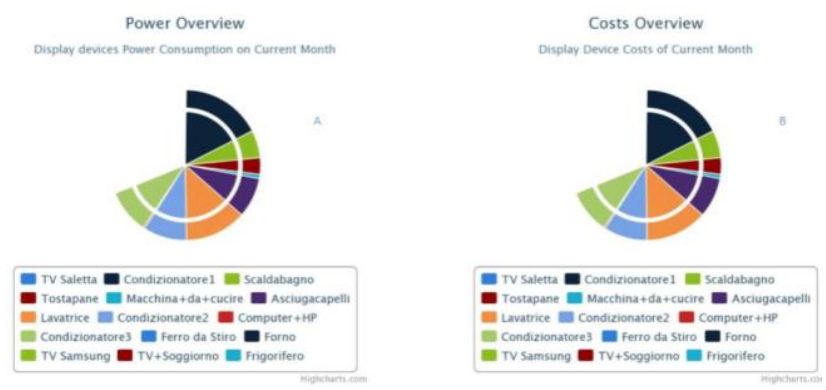

Figure 11 Pie Chart A about Power Consumption Overview, Pie Chart B about Costs Overview.

Before starting the monitoring of a given user, it is very important to characterize it by the historical data taken by the electricity bills. Hereinafter, we will report the results concerning the user that is now under monitoring (user A).

User $A$ has a supply contract with power committed to 3 $\mathrm{kW}$. During the first step of user's characterization, user $A$ communicates the list of appliances connected to the main power supply. In Table 2, the various appliances, grouped by category, of user A' house are placed.

Table 2 User appliances Characterization

\begin{tabular}{|l|l|l|l|}
\hline & \multicolumn{3}{|c|}{ Category } \\
\cline { 2 - 4 } & Light & Appliances & $\begin{array}{l}\text { Computer / } \\
\text { entertainment }\end{array}$ \\
\hline $\begin{array}{l}\text { Total } \\
\text { number }\end{array}$ & 7 & 10 & 7 \\
\hline $\begin{array}{l}\text { Global } \\
\text { Power (W) }\end{array}$ & 271 & 14073 & 666 \\
\hline
\end{tabular}


The user has entered with an energy trader a contract that provides different tariff schemes, in this case a time-ofuse tariff has been agreed.

Actually the Italian Authority for Electricity and Gas (AEEG) approved the entry into force of a mandatory Time-of-Use (ToU) tariff among residential customers subject to the universal supply regime in Italy from July 1st 2010. It provides for variable electricity prices during the day: the price is higher during "peak hours" (the hours between 8 am and 7 pm on working days, also called F1 time slots) and lower during "off-peak hours" (all the remaining hours, also called F2 and F3 time slots, which basically comprise nights and weekends). The AEEG established a 18-months transition period (until December 31st 2011): during such a period the price difference between peak and off-peak hours was limited (transitional ToU tariff) while, starting from January 1st 2012, it has become larger (final ToU tariff), based on the actual electricity market prices. ToU tariff is more convenient than the flat tariff only if more than $2 / 3$ (i.e. 66.67\%) of the total consumption occurs during off-peak hours: such value represents an "indifference threshold".

It is worth specifying that average consumption shift is only $1 \%$, the main two factors that may have prevented a larger consumption shift are: a) rather limited price difference between peak and off-peak hours; b) other components of the final price are not time-dependent the variation on the final price between peak and off-peak hours was even lower. In our case there are three time slots (F1-F2-F3), and the electricity bill depends on the electricity usage habits, i.e. during which hours of the day, and in which day of the week, the appliances are used. Figure 12 shows the F1, F2, F3 daily and weekly time slots (F1 takes about $38 \%$ of hours in a week, whereas F2 takes about the $21 \%$ ) and relative price. On this regard, it is worth noticing that the price of electricity in F1 is about $30 \%$ greater than the one in $\mathrm{F} 3$.

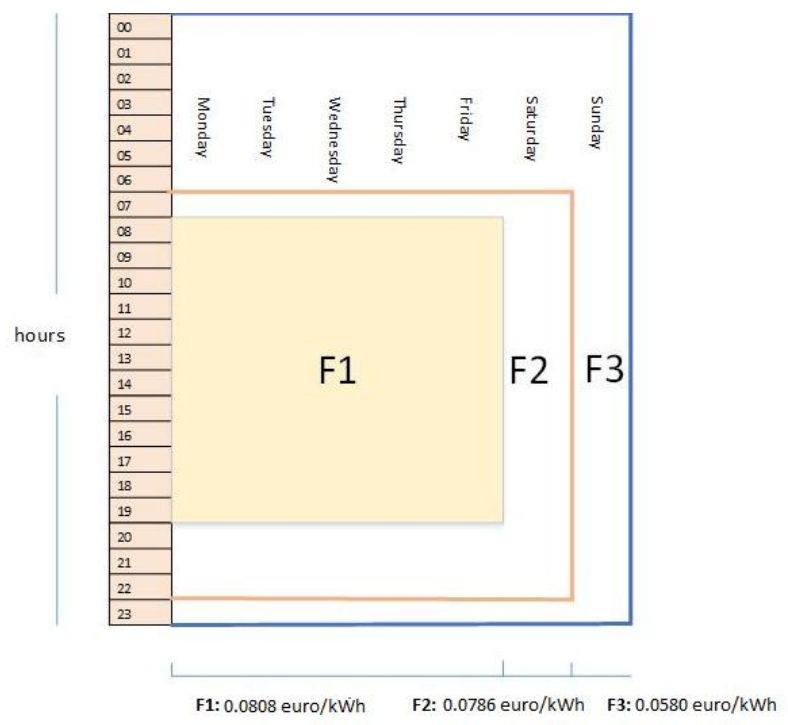

Figure 12 Time of use tariffs, F1, F2, F3: weekly time slots and relative prices.
Starting from the data reported in the electricity bills of an year, some information about energy behavior of the customer under study can be drawn. For example both cooling and heating of the house are based on electrical appliances (e.g. heat pumps), so the electricity demand increases during winter and summer and decreases in the other seasons. Figure 13 shows a bar graph with the monthly average daily demands, and the electricity usage habits over the year is very evident.

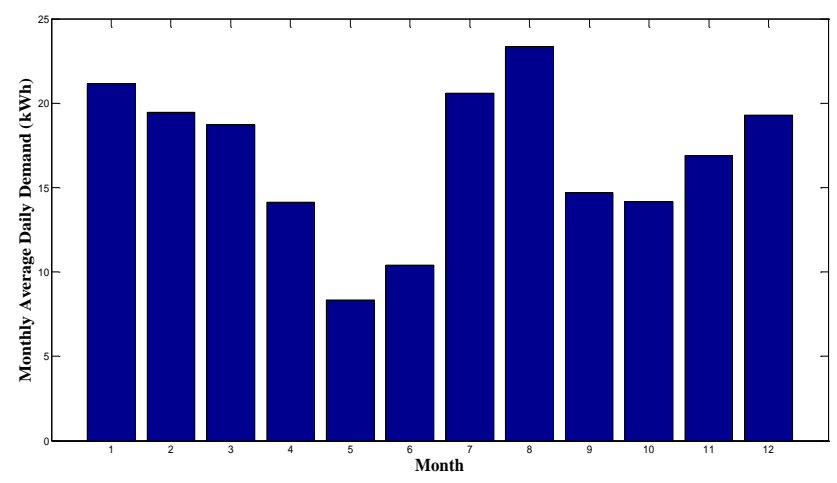

Figure 13 Monthly Average Daily Demand.

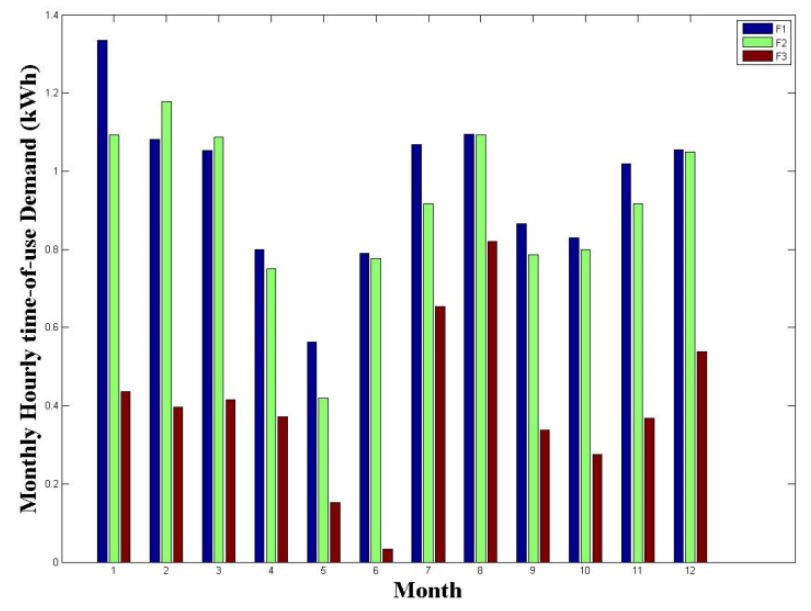

Figure 14 Monthly Hourly time of use Demand

Information reported in Figure 13 is not sufficient to understand how the user behaves respect with his electricity demand during a day and a week, so in Figure 14 the monthly hourly time-of-use demand divided by time slots is reported. It is clear that the demand is greatly concentrated in F1 and F2 slots whereas the nocturnal hours are much less used.

\section{Conclusions}

In this paper a domestic energy monitoring system based on a NIALM algorithm has been presented. The system is non-invasive for the user as it is based on a single point of measurement: a disaggregation algorithm allows to obtain the load of some user's appliances. 
The proposed disaggregation algorithm is simple as it is based on a basic and straightforward signature (i.e. rated active power and power factor), as a consequence the results of this algorithm has to be corrected by means of the user interaction (i.e. feedback)

A software architecture has been designed in order to process all of the data at service provider's side. User information are presented through a user-friendly Web interface; this interface also gathers the user feedback which is needed to improve the efficiency of the disaggregation algorithm. Currently, the proposed research is focusing on the impact of the feedback on the minimization of the error of disaggregation.

The next step in the on field test is to enlarge the number of monitored users in such a way to perform a comparisons with users with similar characteristics. The results of this comparison, shown to the web users, could induce a 'positive competition' in electric demand reduction.

Finally, in the context of Smart Grids, it would be interesting to analyze a domestic user with a photovoltaic (PV) power plant; on this regard forecast methods for both PV production and electric demand could be used to predict them in the next 24 hours, with the aim of improving the predictability of energy exchanges with the network. Forecast algorithms can be also used to predict the preferences of the users in using the home appliances, in order to identify which appliances are going to be used by the user and at what time of the day.

\section{Acknowledgements.}

This work is developed under the SEEE project funded by the POR FESR Sicilia 2007-2013 program.

\section{References}

[1] Organisation for Economic Co-Operation and Development (OECD),"'Smart sensor Networks: Technologies and Applications for Green Growth, December 2009.

[2] Ramchurn, P. Vytelingum, A. Rogers and N. Jennings, "Putting the "smarts" into the smart grid: A grand challenge for artificial intelligence." CACM (2011).

[3] M. Berges et al., Training load monitoring algorithms on highly submetered home electricity consumption data. Tsinghua Science \& Technology, vol. 13, 2008, pp. 406-411.

[4] ABB Group R\&D and Technology, Smart grids. ABB Rewiew, Jenuary 2010.

[5] M. Berges et al., Learning systems for electric consumption of buildings. Proceedings of the 2009 ASCE International Workshop on Computing in Civil Engineering, June 24-27, 2009, Austin, USA, pp. 1-10.

[6] S.N. Patel et al., At the flick of a switch: Detecting and classifying unique electrical events on the residential power line. Proceedings of $9^{\text {th }}$ International Conference on Ubiquitous Computing, Springer-Verlag, Sept. 2007, pp. 271-288.I.S.

[7] T.R. Sharp, Nonintrusive load monitoring systems: Considerations for use and potential applications. US Department of Energy, Office of Scientific and Technical Information, ACEEE Journal, 1994.

[8] G.W. Hart, Nonintrusive appliance load monitoring. Proceedings of the IEEE Computer Applications in Power, vol. 80 (12), Dec. 1992, pp. 1870-1891
[9] G.M.Tina,S.Gagliano,V.A.Amenta, Non Intrusive Load Monitoring Techniques for Energy Emancipation of Domestic Users. International Conference, CISBAT, Lausanne, 4-6 September 2013.

[10] J. Liang, S.K.K. Ng, G. Kendall, J.W.M. Cheng, Load signature study- part I: basic concept, structure, and methodology, IEEE Trans. Power Deliv. 25 (2), (2010) 551-559

[11] G.M.Tina,S.Gagliano,V.A.Amenta, "Web interactive non intrusive load disaggregation system for energy consumption awareness", National Conference AEIT, Mondello, 3-5 October 2013.

[12] Dominik Egarter, Anita Sobe, and Wilfried Elmenreich,"Evolving Non Intrusive Load Monitoring", Procedings of the 15 European Conference on the Applications of Evolutionary and bio-inspired Computation, Vienna, Austria.

[13] G.M.Tina,V.A.Amenta, "Consumption awareness for energy savings: NIALM algorithm efficiency evaluation", IREC ,International Renawble Energy Congress , Hammamet, 25-27 March 2014.

[14] http://ec.europa.eu/energy/efficiency/index_en.htm 